\title{
Aquablation for Benign Prostatic Hyperplasia in Large Prostates (80-150 cc): 1-Year Results
}

\author{
Naeem Bhojani, Mohamed Bidair, Kevin C. Zorn, Andrew Trainer, Andrew Arther, \\ Eugene Kramolowsky, Leo Doumanian, Dean Elterman, Ronald P. Kaufman, \\ James Lingeman, Amy Krambeck, Gregg Eure, Gopal Badlani, Mark Plante, Edward Uchio, \\ Greg Gin, Larry Goldenberg, Ryan Paterson, Alan So, Mitch Humphreys, Steven Kaplan, \\ Jay Motola, Mihir Desai, and Claus Roehrborn
}

OBJECTIVE

METHODS

RESULTS

CONCLUSION
To report 12-month safety and effectiveness outcomes of the Aquablation procedure for the treatment of men with symptomatic benign prostatic hyperplasia $(\mathrm{BPH})$ and large-volume prostates. One hundred and one men with moderate-to-severe BPH symptoms and prostate volumes of 80 $150 \mathrm{cc}$ underwent a robotic-assisted Aquablation procedure in a prospective multicenter international clinical trial. Functional and safety outcomes were assessed at 12 months postoperatively. Mean prostate volume was $107 \mathrm{cc}$ (range 80-150). Mean operative time was 37 minutes and mean Aquablation resection time was 8 minutes. The average length of hospital stay following the procedure was 1.6 days. Mean International Prostate Symptom Score improved from 23.2 at baseline to 6.2 at 12 months $(P<.0001)$. Mean International Prostate Symptom Score quality of life improved from 4.6 at baseline to 1.3 at 12 -month follow-up $(P<.0001)$. Significant improvements were seen in $Q \max (12$-month improvement of $12.5 \mathrm{cc} / \mathrm{sec}$ ) and postvoid residual (drop of $171 \mathrm{cc}$ in those with postvoid residual $>100$ at baseline). Antegrade ejaculation was maintained in $81 \%$ of sexually active men. No patient underwent a repeat procedure for BPH symptoms. There was a $2 \%$ de novo incontinence rate at 12 months, and 10 patients did require a transfusion postoperatively while 5 required take back fulgurations. At 12 months, prostate-specific antigen reduced from $7.1 \pm 5.9 \mathrm{ng} / \mathrm{mL}$ at baseline to $4.4 \pm 4.3 \mathrm{ng} / \mathrm{mL}$.

The Aquablation procedure is demonstrated to be safe and effective in treating men with large prostates $(80-150 \mathrm{cc})$ after 1 year of follow-up, with an acceptable complication rate and without a significant increase in procedure or resection time compared to smaller sized glands. ClinicalTrials.gov number, NCT03123250. UROLOGY 129: 1-7, 2019. (C) 2019 The Authors. Published by Elsevier Inc.
Conflict of Interest: Mihir Desai, Mo Bidair, and Eugene Kramolowsky are consultants for PROCEPT BioRobotics. Mihir Desai is also a consultant with Auris Surgical. Kevin Zorn and Naeem Bhojani have been paid for a training session at AUA 2018. No other author has a conflict of interest with PROCEPT BioRobotics.

Funding: PROCEPT BioRobotics Corporation.

From the University of Montreal Hospital Center, Université de Montréal, Montreal, Quebec, Canada; the San Diego Clinical Trials, San Diego, CA; the Adult Pediatric Urology $\mathcal{E}$ Urogynecology, P.C., Omaha, NE; the Virginia Urology, Richmond, VA; the University of Southern California, Institute of Urology, Los Angeles, CA; the University of Toronto - University Health Network, Toronto, Canada; the Albany Medical College, Albany, NY; the Indiana University Health Physicians, Indianapolis, IN; the Urology of Virginia, Virginia Beach, VA; the Wake Forest School of Medicine, WinstonSalem, NC; the University of Vermont Medical Center, Burlington, VT; the VA Long Beach Healthcare System, Long Beach, CA; the University of British Columbia, Vancouver, Canada; the Mayo Clinic Arizona, Scottsdale, AZ; the Icahn School of Medicine at Mount Sinai, New York, NY; and the UT Southwestern Medical Center, Department of Urology, University of Texas Southwestern, Dallas, TX

Address correspondence to: Naeem Bhojani, M.D., F.R.C.S.C., University of Montreal, Division of Urology, 900 St. Denis, R08.474, Montreal, Quebec H2X OA9, Canada. E-mail:Naeem.bhojani@gmail.com

Submitted: March 5, 2019, accepted (with revisions): April 30, 2019

(C) 2019 The Authors. Published by Elsevier Inc.

This is an open access article under the CC BY-NC-ND license.

(http://creativecommons.org/licenses/by-nc-nd/4.0/)
7 he first transurethral resection of the prostate (TURP) was performed by Maximilian Stern in 1926. Over 90 years later, it is still considered by some to be the gold standard treatment for benign prostatic hyperplasia (BPH) although rarely can it be applied to all sizes of prostate glands. The past several decades have witnessed many innovative technologies developed for BPH and incorporated into the daily practice of urology. Two of these notable technologies are ultrasound and robotics. Ultrasound real-time guidance is being used more and more in the field of medicine and makes the application of interventions specific and precise. Additionally, robotic execution has become the treatment of choice for localized prostate cancer and has demonstrated reproducible and excellent outcomes. The Aquablation procedure is a novel technology that integrates both real-time ultrasonic imaging with robotically executed surgeon-guided high-velocity waterjet ablation to precisely resect prostatic tissue. High- 
pressure water jet technology is already used in the metal, ceramic, and glass industries and has been described for tissue-specific liver resection ${ }^{1}$ and bladder tumors. ${ }^{2}$ In prostatic disease, a blinded randomized trial (WATER) of the Aquablation procedure vs TURP in 30-80 cc prostates demonstrated that the Aquablation procedure has similar efficacy when compared to TURP but with considerably shorter resection times, lower risk of sexual dysfunction, and overall reduced morbidity. ${ }^{3}$ In the WATER trial, a subgroup analysis of larger prostate glands (50-80 cc), demonstrated a superior symptom-reduction measured by International Prostate Symptom Score (IPSS) for the Aquablation procedure compared to TURP. Additionally, observations during the WATER study indicated that the Aquablation procedure time was fast (33 minutes) and independent of prostatic volume. This prompted a prospective multicenter study to evaluate the safety and efficacy of treating larger volume prostate glands.

The purpose of this report is to detail the12-month safety and efficacy data from WATERII, a prospective multicenter trial of the Aquablation procedure in men with symptomatic BPH and prostate volumes between 80 and $150 \mathrm{cc}$.

\section{METHODS}

\section{Trial Design and Participants}

WATERII (NCT03123250) is a prospective, multicenter, international clinical trial of the Aquablation procedure for the treatment of LUTS due to BPH in men 45-80 years of age with a prostate volume between 80 and $150 \mathrm{cc}$ as measured by preoperative transrectal ultrasound. The study was sponsored by the device manufacturer. Eligibility criteria were as follows: baseline IPSS $^{4} \geq 12$, a maximum urinary flow rate (Qmax) $<15 \mathrm{~mL} / \mathrm{s}$, a serum creatinine $<2 \mathrm{mg} / \mathrm{dL}$, a history of inadequate or failed response to medical therapy and mental capability and willingness to participate in the study. Men were excluded if they had body mass index $\geq 42 \mathrm{~kg} / \mathrm{m}^{2}$, a history of prostate or bladder cancer, clinically significant bladder calculus or bladder diverticulum, active infection, previous urinary tract surgery, urinary catheter use daily for 90 or more days, chronic pelvic pain, diagnosis of urethral stricture, meatal stenosis or bladder neck contracture, use of anticholinergic agents, and other general conditions that could prevent adequate study follow-up. Patients with prior prostate surgery were not excluded. Men with urinary retention were excluded if the catheter was in place for more than 90 days. Institutional Review Board/Ethics Committee approval prior to study-related consent was obtained at each individual site. In the United States, the study was run under investigational device exemption from US Food and Drug Administration.

At both baseline and at selected follow-up visits, the following questionnaires were completed: IPSS, Incontinence Severity Index, Pain Intensity Scale, International Index of Erectile Function (IIEF-15 $)$, the Male Sexual Health Questionnaire $\left(\mathrm{MSHQ}-\mathrm{EjD}^{6}\right)$, uroflowmetry, and postvoid residual volume measurements. Serum prostate-specific antigen (PSA) was performed at baseline and at 6 and 12 months. Transrectal ultrasound prostate size measurements were performed preoperatively and at 3 months postoperatively. Standard laboratory tests (blood count/serum chemistries) were performed at baseline and prior to hospital discharge.

The Aquablation procedure was performed using the AQUABEAM System (PROCEPT BioRobotics, Redwood City, CA), as described previously. ${ }^{7}$ Briefly, after induction of general or spinal anesthesia, a 24F handpiece was inserted into the prostatic urethra and secured into place using a bed-mounted arm. Under real-time transrectal ultrasound (BK Medical, Peabody, MA) guidance, the surgeon defined the target anatomic resection contour on a computer console. Resection contours were drawn to avoid damage to the bladder neck, ejaculatory ducts, and urinary sphincter. Tissue was then ablated under robotic execution utilizing a high-velocity waterjet that moves in a controlled manner from the bladder to the verumontanum. For larger prostates, the Aquablation procedure typically required 2 passes of the AQUABEAM probe.

After the Aquablation procedure, the bladder was manually irrigated to remove residual prostate tissue and blood clots. Hemostasis was achieved via low-pressure tamponade with a Foley balloon catheter inflated to $40-80 \mathrm{cc}$ of saline either at the bladder neck or within the prostatic fossa, followed by continuous bladder irrigation. The study's primary safety and efficacy endpoints were calculated at 3 months (previously reported ${ }^{8}$ ) but we report herein outcomes to 12 months.

\section{Data Monitoring}

All study data were collected using an electronic data capture system. Study data were $100 \%$ source-verified by study monitors. Adverse events were collected throughout follow-up and evaluated by an independent clinical events committee of 3 practicing urologists.

\section{Study Endpoints and Statistical Analysis}

The study's previously reported primary efficacy endpoint was the change in total IPSS score from baseline to 3 months. The study's primary safety endpoint was the proportion of subjects with adverse events rated as possibly, probably, or definitely related to the study procedure classified as Clavien-Dindo (CD) Grade 2 or higher or any Grade 1 event resulting in persistent disability (eg, ejaculatory disorder, erectile dysfunction, or permanent incontinence) evidenced through 3 months post-treatment. This primary safety endpoint was $<65 \%$ of patients. Longer term changes in symptom scores and uroflow measures (all continuous outcomes) were assessed using either $t$ tests or repeated measures analysis of variance. The change in MSHQ$\mathrm{EjD}$ at 3 months was considered to represent relatively preserved ejaculatory function if the decrease was noninferior to -4 points. Similarly, preserved erectile function was assumed if the IIEF-5 (SHIM) score decrease at 3 months was noninferior to -6 points. The noninferior thresholds for the sexual function outcomes were selected as an estimate to detect a clinically meaningful change. Exact binomial methods were used to calculate confidence intervals for proportions. All statistical analysis was performed using R. ${ }^{9}$

\section{RESULTS}

One hundred and one subjects were enrolled and treated at 16 sites (24 surgeons) between September and December 2017. Three sites were in Canada and the remainder were in the United States. Twelve month follow-up was completed by 97 of $101(97 \%)$ of subjects (Supplementary Fig. 1). 
Table 1. Baseline characteristics $(n=101)$

\begin{tabular}{|c|c|}
\hline Characteristic & Statistic \\
\hline Age, years, mean (SD), range & $67.5(6.6), 52-79$ \\
\hline Body mass index, mean (SD), range & 28.4 (4.2), $22-41$ \\
\hline \multicolumn{2}{|l|}{ Race } \\
\hline Asian & $5(5.0 \%)$ \\
\hline Black & $6(5.9 \%)$ \\
\hline White & $88(87.1 \%)$ \\
\hline Other & $2(2.0 \%)$ \\
\hline \multicolumn{2}{|l|}{ Ethnicity } \\
\hline Hispanic or Latino & $9(8.9 \%)$ \\
\hline Non-Hispanic or Latino & $92(91.1 \%)$ \\
\hline $\begin{array}{l}\text { Prostate specific antigen, g/dL; } \\
\text { mean (SD), range }\end{array}$ & 7.1 (5.9), 0.34-29 \\
\hline $\begin{array}{l}\text { Use of catheters in } 45 \mathrm{~d} \text { prior to } \\
\text { enrollment }\end{array}$ & $14(14.3 \%)$ \\
\hline $\begin{array}{l}\text { Prostate size (TRUS), cc; mean } \\
\text { (SD), range }\end{array}$ & 107.4 (22.1), 80-150 \\
\hline Middle lobe & $84(83.2 \%)$ \\
\hline Intravesical component & $81(96.4 \%)$ \\
\hline $\begin{array}{l}\text { Intravesical protrusion, } \mathrm{mm} \text {; } \\
\text { mean (SD) }\end{array}$ & $1.8(0.8)$ \\
\hline \multicolumn{2}{|l|}{ Baseline questionnaires } \\
\hline IPSS score, mean (SD), range & $23.2(6.3), 12-35$ \\
\hline IPSS QOL, mean (SD), range & 4.6 (1.0), 2-6 \\
\hline Sexually active, N (\%) [MSHQ-EjD] & $77(76.2 \%)$ \\
\hline MSHQ-EjD*, mean (SD), range & 8.1 (3.9), 1-15 \\
\hline SHIM*, mean (SD), range & $15.1(7.4), 2-25$ \\
\hline
\end{tabular}

* Sexually active men only.

Baseline patient characteristics are summarized in Table 1. Mean age was 68 years (63-72) and baseline IPSS was 23 (1235). Mean prostate volume was $107 \mathrm{cc}(80-150)$. A median lobe was present in $83 \%$ of cases with an average intravesical prostatic protrusion distance of $1.8 \mathrm{~cm}(0.7-6.8)$. Study procedures were performed under general anesthesia in $18 \%$ and spinal anesthesia in $82 \%$ of cases.

Mean operative time (handpiece placement to urinary catheter placement) was 37 minutes (15-97 minutes) and mean Aquablation resection time was 7.8 minutes (2.5-17 minutes). A single Foley balloon catheter $(22 \mathrm{Fr}-24 \mathrm{Fr})$ was placed in the bladder under mild tension for the sole means of hemostasis in 98 $(97.0 \%)$ cases with bladder traction maintained for an average of 18 hours (2.1-50 hours). In the other 3 cases, the Foley catheter balloon was deployed in the prostatic fossa for direct tamponade and used for an average of 15 hours (0.55-25 hours; Supplementary Table 1). Forty-five percent of patients required postoperative pain medication (narcotics) and 23\% required bladder spasm medication. No subject underwent post-Aquablation cautery or treatment for hemostasis at the time of the primary procedure. Postoperatively, 59\% of subjects were discharged within 1 day and the mean length of stay was 1.6 days (0-6 days). Two patients went home the same day of surgery. Most patients (68\%) were discharged home with a catheter; which was removed on average 4 days (0.7-30 days) postAquablation procedure. Hemoglobin levels decreased from a mean of 14.8 at baseline to 11.9 prior to discharge (drop of $2.9 \mathrm{~g} / \mathrm{dL}, P<.0001)$. Overall bleeding requiring transfusions occurred in 10 patients ( 2 of them also requiring return to the operating room for fulguration) and 3 additional patients who required fulguration only (no transfusions).

Mean (SD) IPSS improved from $23.2(6.3)$ at baseline to 6.2 (5.0) at 12 months (a 17-point improvement, 2, $P<.0001$,
Fig. 1). The 12-month IPSS scores were independent of baseline IPSS. IPSS QOL decreased from 4.6 (1) at baseline to 1.3 (1.5) at 12 months $(P<.0001)$. Maximum urinary flow rate increased from 8.7 (3.4) to $21.1 \mathrm{cc} / \mathrm{sec}(12.1$; an improvement of $12.5 \mathrm{cc} / \mathrm{sec}$ (12.4), $P<.0001)$ and postvoid residual urinary volume decreased from $131 \mathrm{~mL}(125)$ at baseline to $51 \mathrm{~mL}(67)$ at 12 months. There was a $38 \%$ reduction in serum PSA from an average of $7.1 \pm 5.9 \mathrm{ng} / \mathrm{mL}$ at baseline to $4.4 \pm 4.3 \mathrm{ng} / \mathrm{mL}$ at 12 months.

At baseline, 77 (76\%) subjects were sexually active. Among subjects reporting sexual activity at baseline and at follow-up study visits, the mean total MSHQ-EjD decreased slightly from 8.1 (95\% confidence interval 7.2-9.0) at baseline to $7(5.8-8.3)$ at 3 months and $6.6(5.4-7.9)$ at 12 months (Fig. 2). The 3 and 12 month decreases ( -2 points) met the study's noninferiority hypothesis $(P=.0026)$.

In subjects who were sexually active at both baseline and at the follow-up study visit, IIEF-5 (SHIM) scores were unchanged from baseline (15.1) to 12-month follow-up (16.3), Figure 2. At 3 months, the decrease in IIEF-5 score was less than 6 points $(P<.0001)$, meeting the study's predetermined threshold. IIEF-15 scores showed no major changes in any category (Supplementary Fig. 2). No subject reported any de novo erectile dysfunction.

Sixteen subjects entered the trial using a urinary catheter routinely within 45 days prior to treatment. At the 3-month visit, no subject was using a catheter routinely.

The primary safety endpoint, defined as CD Grade 2 or higher or any Grade 1 event resulting in persistent disability (eg, ejaculatory disorder, erectile dysfunction, or permanent incontinence), at 3 months occurred in $45.5 \%$ of men, which met the study design goal of less than $65 \%(P<0.0001)$. Ejaculatory dysfunction occurred in $19 \%$ of sexually active men. Therefore, $81 \%$ of sexually active men maintained antegrade ejaculation. Of the $5(5 \%)$ patients with incontinence requiring the use of a pad at 6 months, only $3(3 \%)$ required a pad at 12 months. There was 1 additional patient that had an artificial urinary sphincter inserted for persistent stress incontinence (CD3). Of the 4 incontinence subjects, 2 of them had incontinence symptoms at baseline. A nonhierarchical breakdown of CD events at 12 months resulted in $22 \%$ grade $2,14 \%$ grade 3 , and $5 \%$ grade 4 events (Table 2), the majority of which occurred within the first month after the procedure. There were 5 patients that had grade 4 events: (1) a bleeding event requiring a transfusion on the same day as surgery, (2) a bleeding event requiring a transfusion on the same day as surgery and the patient experienced bradycardia, (3) a subject had a stroke the day after the procedure (cause of the stroke was not determined) subsequently the subject went into multiorgan system failure but fully recovered, (4) a subject had complete heart block 8 days after the procedure, and (5) 1 subject developed chest pain and myocardial infarction from a left main coronary artery occlusion and underwent repeated angioplasty but fully recovered. No strictures, no retention, no secondary procedures for tissue removal or late bleeding events (>30 days postop) was otherwise required during the 12 months of follow-up.

\section{DISCUSSION}

This prospective multicenter trial demonstrates that the Aquablation procedure of the prostate is a practical, easily reproducible and clinically effective option for the treatment of large sized prostate glands $(80-150 \mathrm{cc})$ up to at 

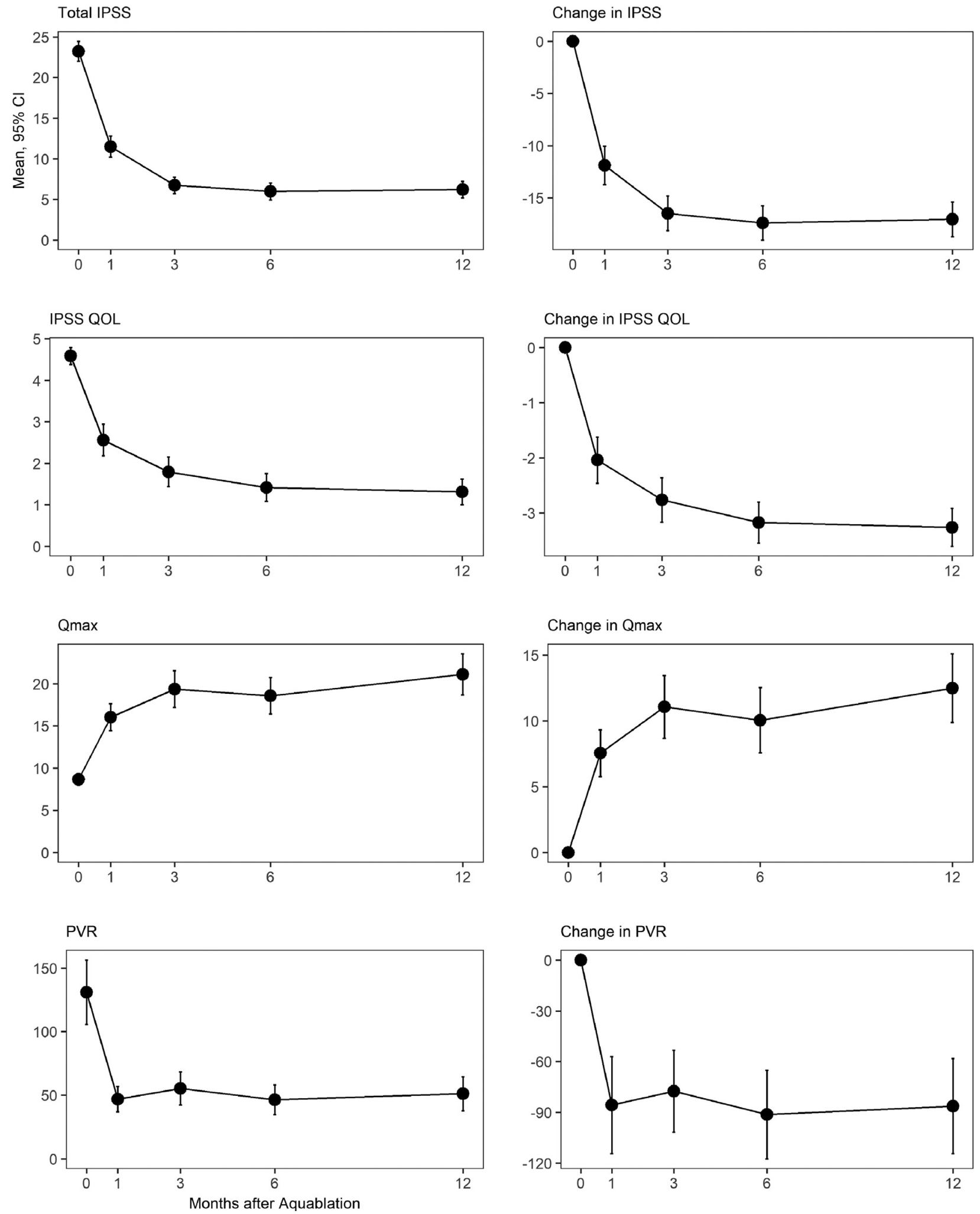

Figure 1. Improvement in parameters after Aquablation: (A) IPSS; (B) IPSS quality of life (QOL); (C) Maximum urinary flow rate (cc/sec); (D) Postvoid residual (cc).

least 1 year. Furthermore, there are several noteworthy findings. All cases 101 of 101 (100\%) were successfully completed for prostates $>100 \mathrm{cc}$ on average and over $80 \%$ with a significant median lobe. Additionally, no secondary procedures for $\mathrm{BPH}$ recurrence have been required.
Operating room time efficiency, an important advantage noticed during the first WATER study where procedure (33 minutes) and resection (4 minutes) times were only marginally impacted by prostate volume, remained favorable in this study with larger prostate glands. Total 

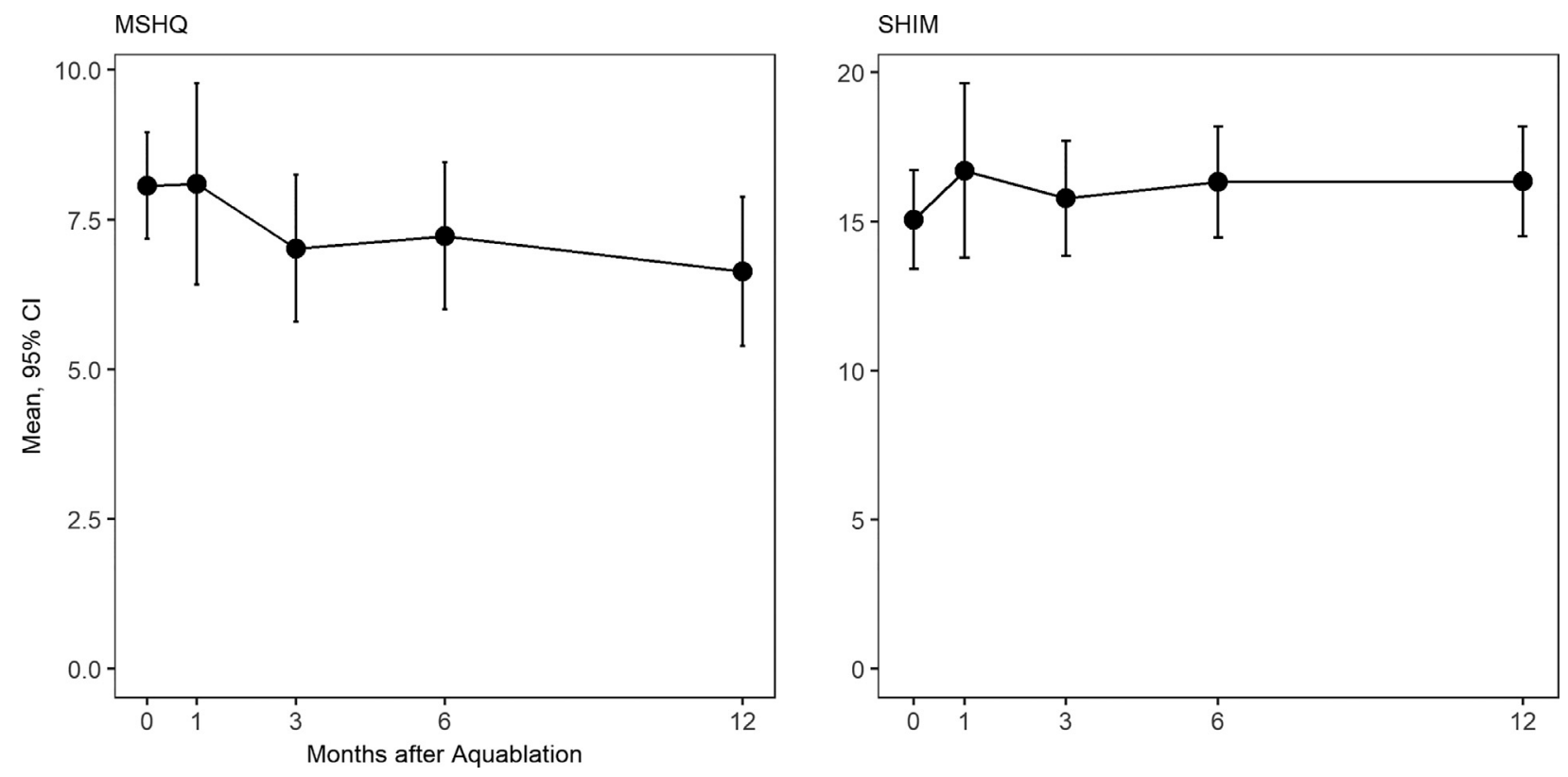

Figure 2. Sexual function outcomes in sexually active men. (A) MSQH-EjD; (B) SHIM.

operative time and total resection time was 37 and 8 minutes respectively which is considerably shorter than the average time to perform a $100 \mathrm{cc}$ prostate via open prostatectomy $\left(95\right.$ minutes $\left.^{10}\right)$, holmium laser enucleation of the prostate (HoLEP; 91 minutes $^{11}$ ), or photoselective vaporization of the prostate (PVP; 93 minutes ${ }^{12}$ ).

The Aquablation procedure continued to be efficacious in treating bladder outlet obstruction in patients with large prostates at 12 months with an average IPSS reduction of 17, QoL reduction of 3, and Qmax increase of $12 \mathrm{~mL} / \mathrm{s}$. These clinical improvements are comparable to those found with HoLEP (IPSS decline of 14.7 and Qmax increase of $14.3 \mathrm{~mL} / \mathrm{s}$ ) ${ }^{13}$ and PVP (IPSS decline of 17 and Qmax increase of $10 \mathrm{~mL} / \mathrm{s}){ }^{12}$ Additionally, the $8 \%$ urinary incontinence rate at 1 month for the Aquablation procedure reduced to $4 \%$ at 12 months which is less than that reported for HoLEP $\left(4.9 \%-12.5 \%{ }^{13-15}\right)$ and comparable to open prostatectomy $\left(3 \%-9 \%{ }^{16,17}\right)$ and

Table 2. Distribution of events at month 12 categorized by Clavien-Dindo grades by group rated as possibly, probably, or definitely related to the procedure/device

\begin{tabular}{|c|c|c|c|c|}
\hline CD Grade* & Term & Events & Subjects & Rate \\
\hline \multirow[t]{10}{*}{2} & Bleeding & 8 & 6 & $5.9 \%$ \\
\hline & Cardiac & 1 & 1 & $1.0 \%$ \\
\hline & Dysuria & 2 & 2 & $2.0 \%$ \\
\hline & Infection & 2 & 2 & $2.0 \%$ \\
\hline & Other & 2 & 2 & $2.0 \%$ \\
\hline & Pain & 1 & 1 & $1.0 \%$ \\
\hline & Urinary frequency & 2 & 2 & $2.0 \%$ \\
\hline & Urinary tract infection & 7 & 6 & $5.9 \%$ \\
\hline & Urinary urgency & 1 & 1 & $1.0 \%$ \\
\hline & Total & 26 & 22 & $21.8 \%$ \\
\hline \multirow[t]{8}{*}{3} & Bleeding & 7 & 6 & $5.9 \%$ \\
\hline & Dysuria & 1 & 1 & $1.0 \%$ \\
\hline & Meatal stenosis & 4 & 3 & $3.0 \%$ \\
\hline & Urethral stricture & 1 & 1 & $1.0 \%$ \\
\hline & Urinary retention & 1 & 1 & $1.0 \%$ \\
\hline & Urinary incontinence & 1 & 1 & $1.0 \%$ \\
\hline & Urinary urgency & 1 & 1 & $1.0 \%$ \\
\hline & Total & 16 & 14 & $13.9 \%$ \\
\hline \multirow[t]{5}{*}{4} & Bleeding & 2 & 2 & $2.0 \%$ \\
\hline & Cardiac & 2 & 2 & $2.0 \%$ \\
\hline & Cerebrovascular accident & 1 & 1 & $1.0 \%$ \\
\hline & Multisystem organ failure & 1 & 1 & $1.0 \%$ \\
\hline & Total & 6 & 5 & $5.0 \%$ \\
\hline
\end{tabular}

* CD Grade 2 = complication requiring pharmacological treatment with drugs other than such allowed for grade I complications. Includes blood transfusions and total parenteral nutrition. Grade $3=$ complication requiring surgical, endoscopic or radiological intervention. Grade 4 = life-threatening complication (including CNS complications) requiring intensive care. 
TURP $\left(2 \%{ }^{18}\right)$. Since 2 of the 4 patients had incontinence at baseline, the de novo incontinence rate at 1 year for Aquablation is $2 \%$. Furthermore, the rate of dysuria was also extremely low at only $2 \%$ due to the absence of cautery and/or laser energy.

At 12 months, the PSA dropped from a baseline of 7.1 to $4.4 \mathrm{ng} / \mathrm{mL}$ (38\% reduction). This PSA reduction is slightly less than for PVP using the newer Greenlight XPS-180 system $^{12}$ which demonstrated a PSA drop of $49 \%$ in men with prostate glands between 107 and 150 cc. Although the PSA drops we observed after Aquablation were smaller than those observed after HOLEP $(>80 \%),{ }^{19-21}$ rates of urge urinary incontinence and retrograde ejaculation are significantly higher with HOLEP $\left(70 \%-80 \%{ }^{22-25}\right)$.

After 12 months, $81 \%$ of the sexually active men in this study maintained their antegrade ejaculatory function, the mean MSHQ-EjD score dropped by only -1.4 , and the SHIM score dropped by 0.1 . Thus, while the maintenance of antegrade ejaculation dropped from our randomized trial where prostate volumes were much smaller, this to our knowledge far exceeds the rate of antegrade ejaculation compared to any other surgical technique for large prostate glands $(>100 \mathrm{cc})$. The reason behind this is most likely due to the ultrasound guidance and robotically executed nature of the procedure which allows for precise treatment and ejaculatory duct and bladder neck preservation. In addition, a "butterfly" technique is used near the ejaculatory ducts which further maintain the ejaculatory ducts and likely helps with the maintenance of antegrade ejaculation.

Finally, the safety of the procedure has been maintained up to 12 months and no patient has undergone a secondary procedure for $\mathrm{BPH}$. The Overall CD Grades 2-4 complications were previously reported at 22\%, $14 \%$, and 5\%, respectively. Bleeding after the Aquablation procedure and prior to discharge that required a transfusion was observed in 10 patients. This is much lower compared to the hemorrhage rate for a simple prostatectomy (range $12 \%-29 \%$ ) but higher than that reported with HOLEP (range 0\%-4\%). ${ }^{26,27}$ Although prostates glands in this study were larger than the previous Aquablation trial, bleeding was only seen in a limited number of patients. Adequate hemostasis was achieved using manual irrigation post procedure followed by catheter traction for 15 18 hours on average.

The surgical management of BPH has undergone significant innovations since the advent of the first successful TURP, with the introduction of numerous surgical options and energy modalities. It was established during the WATER trial that the Aquablation procedure was equivalent to TURP for prostate gland sizes from 30 to 80 cc. This study demonstrates that the Aquablation procedure can be applied to large prostate glands from 80 to $150 \mathrm{cc}$. For surgeons who do not perform HoLEP, the current treatment of choice for large prostate glands $(>100$ grams) is open prostatectomy. Compared to open prostatectomy, the Aquablation procedure may provide a significantly better treatment alternative with a very short learning curve, shorter operative time, shorter length of stay, shorter length of catheterization, lower transfusion rates, and reduced morbidity. Therefore, the Aquablation procedure has the unique advantage of being applicable to most prostate sizes with minimal impact on the length of procedure or on the skills, experience, or technique of the treating surgeon. In the present study, the majority of surgeons had no prior experience (average 0.5 cases/surgeon) and the average number of cases in the trial was 4 , thus attesting to a very short learning curve. In comparison, HoLEP requires between 25 and 50 cases to become proficient $^{28}$ and PVP requires up to 100 cases to become proficient. ${ }^{29}$ Another major advantage of the Aquablation procedure is that it is reproducible. The ultrasound live image guidance and robot execution significantly reduce the variability of the procedure. Therefore, the variability of outcomes is accordingly reduced.

Similar results using the AQUABEAM system have recently been published in a prospective cohort from Germany, demonstrating large improvements in IPSS, Qmax, and postvoid residual, along with a $65 \%$ prostate volume reduction measured by TRUS and a low complication rate. ${ }^{30}$

Despite its merits to assess the Aquablation procedure in men with BPH and with significantly larger prostates, this study has limitations worthy of mention. The main limitation is that WATERII trial is a single arm study without a control group preventing direct comparisons with those techniques. Additionally, standardized reporting of events categorized by CD scores was limited in the literature. In addition, surgeon experience with Aquablation is still relatively limited and additional experience will probably improve outcomes. Finally, while the outcomes are promising, longer follow-up will be necessary to confirm these results.

\section{CONCLUSION}

The Aquablation procedure is a safe surgical option in patients with large prostate glands, with durable outcomes at 1 year coupled with fast operative times, short hospitalizations, and the maintenance of antegrade ejaculatory function. There were acceptable complications and transfusion rates reported. The learning curve, even in the setting of large prostate volumes, is remarkably short. The Aquablation procedure has been demonstrated to be an effective and reproducible treatment for $\mathrm{BPH}$ independent of prostate size up to $150 \mathrm{cc}$.

Acknowledgments. The authors would like to acknowledge the research coordinators and patients who participated in this study.

\section{SUPPLEMENTARY MATERIALS}

Supplementary material associated with this article can be found in the online version at https://doi.org/10.1016/ j.urology.2019.04.029. 


\section{References}

1. Baer HU, Stain SC, Guastella T, Maddern GJ, Blumgart LH. Hepatic resection using a water jet dissector. HPB Surg World J Hepatic Pancreat Biliary Surg. 1993;6:189-196. discussion 196-8.

2. Nagele U, Kugler M, Nicklas A, et al. Waterjet hydrodissection: first experiences and short-term outcomes of a novel approach to bladder tumor resection. World J Urol. 2011;29:423-427. https://doi.org/ 10.1007/s00345-011-0653-8.

3. Gilling P, Barber N, Bidair M, et al. WATER: a double-blind, randomized, controlled trial of aquablation $((\mathrm{R}))$ vs transurethral resection of the prostate in benign prostatic hyperplasia. J Urol. 2018;199:1252-1261. https://doi.org/10.1016/j.juro.2017.12.065.

4. Barry MJ, Fowler FJ, O'Leary MP, et al. The American Urological Association symptom index for benign prostatic hyperplasia. The Measurement Committee of the American Urological Association. J Urol. 1992;148:1549-1557. discussion 1564.

5. Rosen RC, Allen KR, Ni X, Araujo AB. Minimal clinically important differences in the erectile function domain of the International Index of Erectile Function scale. Eur Urol. 2011;60:1010-1016. https://doi.org/10.1016/j.eururo.2011.07.053.

6. Rosen RC, Catania J, Pollack L, Althof S, O'Leary M, Seftel AD. Male Sexual Health Questionnaire (MSHQ): scale development and psychometric validation. Urology. 2004;64:777-782. https://doi. org/10.1016/j.urology.2004.04.056.

7. Gilling P, Reuther R, Kahokehr A, Fraundorfer M. Aquablationimage-guided robot-assisted waterjet ablation of the prostate: initial clinical experience. BJU Int. 2016;117:923-929. https://doi.org/ 10.1111/bju. 13358 .

8. Desai M, Bidair M, Bhojani N, et al. WATER II ( $80-150 \mathrm{~mL})$ procedural outcomes. BJU Int. 2018. https://doi.org/10.1111/bju.14360.

9. R Core Team. R: A Language and Environment for Statistical Computing. Vienna, Austria: R Foundation for Statistical Computing; 2016. https://www.R-project.org/.

10. Sorokin I, Sundaram V, Singla N, et al. Robot-assisted versus open simple prostatectomy for benign prostatic hyperplasia in large glands: a propensity score-matched comparison of perioperative and shortterm outcomes. J Endourol. 2017;31:1164-1169. https://doi.org/ 10.1089/end.2017.0489.

11. Monn MF, El Tayeb M, Bhojani N, et al. Predictors of enucleation and morcellation time during holmium laser enucleation of the prostate. Urology. 2015;86:338-342. https://doi.org/10.1016/j.urology. 2015.04.028.

12. Valdivieso R, Hueber P-A, Meskawi M, et al. Multicentre international experience of 532-nm laser photoselective vaporization with GreenLight XPS in men with very large prostates. BJU Int. 2018. https://doi.org/10.1111/bju.14208.

13. Krambeck AE, Handa SE, Lingeman JE. Experience with more than 1,000 holmium laser prostate enucleations for benign prostatic hyperplasia. J Urol. 2013;189(1 Suppl):S141-S145. https://doi.org/ 10.1016/j.juro.2012.11.027.

14. Elmansy HM, Kotb A, Elhilali MM. Is there a way to predict stress urinary incontinence after holmium laser enucleation of the prostate. J Urol. 2011;186:1977-1981. https://doi.org/10.1016/j.juro.2011.06.063.

15. Vavassori I, Valenti S, Naspro R, et al. Three-year outcome following holmium laser enucleation of the prostate combined with mechanical morcellation in 330 consecutive patients. Eur Urol. 2008;53:599-604. https://doi.org/10.1016/j.eururo.2007.10.059.
16. Serretta V, Morgia G, Fondacaro L, et al. Open prostatectomy for benign prostatic enlargement in southern Europe in the late 1990s: a contemporary series of 1800 interventions. Urology. 2002;60: 623-627.

17. Tubaro A, Carter S, Hind A, Vicentini C, Miano L. A prospective study of the safety and efficacy of suprapubic transvesical prostatectomy in patients with benign prostatic hyperplasia. J Urol. 2001;166:172-176.

18. Montorsi F, Naspro R, Salonia A, et al. Holmium laser enucleation versus transurethral resection of the prostate: results from a 2-center, prospective, randomized trial in patients with obstructive benign prostatic hyperplasia. J Urol. 2004;172(5 Pt 1):1926-1929.

19. Elmansy H, Baazeem A, Kotb A, et al. Holmium laser enucleation versus photoselective vaporization for prostatic adenoma greater than $60 \mathrm{ml}$ : preliminary results of a prospective, randomized clinical trial. J Urol. 2012;188:216-221. https://doi.org/10.1016/j.juro.2012.02.2576.

20. Tinmouth WW, Habib E, Kim SC, et al. Change in serum prostate specific antigen concentration after holmium laser enucleation of the prostate: a marker for completeness of adenoma resection. J Endourol. 2005;19:550-554. https://doi.org/10.1089/end.2005.19.550.

21. Desai M, Bidair M, Zorn KC, et al. Aquablation for BPH in Large Prostates (80-150cc): 6-Month Results From the WATER II Trial. BJU Int. 2019. https://doi.org/10.1111/bju.14703.

22. Placer J, Salvador C, Planas J, et al. Effects of holmium laser enucleation of the prostate on sexual function. J Endourol. 2015;29:332339. https://doi.org/10.1089/end.2014.0502.

23. Meng F, Gao B, Fu Q, et al. Change of sexual function in patients before and after Ho:YAG laser enucleation of the prostate. J Androl. 2007;28:259-261. https://doi.org/10.2164/jandrol.106.000372.

24. Elshal AM, Elmansy HM, Elkoushy MA, Elhilali MM. Male sexual function outcome after three laser prostate surgical techniques: a single center perspective. Urology. 2012;80:1098-1104. https://doi.org/ 10.1016/j.urology.2012.08.001.

25. Montorsi F, Naspro R, Salonia A, et al. Holmium laser enucleation versus transurethral resection of the prostate: results from a 2-center, prospective, randomized trial in patients with obstructive benign prostatic hyperplasia. J Urol. 2004;172:1926-1929. https://doi.org/ 10.1097/01.ju.0000140501.68841.a1.

26. Pariser JJ, Pearce SM, Patel SG, Bales GT. National Trends of Simple Prostatectomy for Benign Prostatic Hyperplasia With an Analysis of Risk Factors for Adverse Perioperative Outcomes. Urology. 2015;86:721-726. https://doi.org/10.1016/j.urology.2015.06.048.

27. Gratzke C, Schlenker B, Seitz M, et al. Complications and early postoperative outcome after open prostatectomy in patients with benign prostatic enlargement: results of a prospective multicenter study. J Urol. 2007;177:1419-1422. https://doi.org/10.1016/j.juro.2006.11.062.

28. Kampantais S, Dimopoulos P, Tasleem A, Acher P, Gordon K, Young A. Assessing the learning curve of holmium laser enucleation of prostate (HoLEP). A systematic review. Urology. 2018;120:9-22. https://doi.org/10.1016/j.urology.2018.06.012.

29. Bastard C, Zorn K, Peyronnet B, et al. Assessment of learning curves for 180-W GreenLight XPS photoselective vaporisation of the prostate: a multicentre study. Eur Urol Focus. 2017. https://doi.org/ 10.1016/j.euf.2017.09.011.

30. Bach T, Giannakis I, Bachmann A, et al. Aquablation of the prostate: single-center results of a non-selected, consecutive patient cohort. World J Urol. 2018. https://doi.org/10.1007/s00345-018-2509-y. 